\title{
Agricultural Economics and Social Science
}

http:/www.journals.zu.edu.eg/journalDisplay.aspx?Journalld=1\&queryType=Master

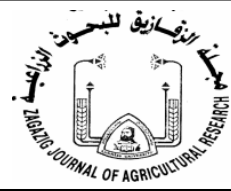

دراسة اقتصادية للمفرخات السمكية البحرية في مصر : دراسة حالة مفـــــ حراز بالإســـماعيلية

$$
\begin{aligned}
& \text { رشا عبد الهادي عبد المنعم نايل1* ـ محمد جابر محمد عامر_ـ علي أحمد ابراهيم² محمد محمد سليمان } 1 \\
& 1 \text { ـ معهد بحوث الاقتصاد الزر اعي ـ مركز البحوث الزر اعية ـ مصر } \\
& \text { 2- قسم الاقتصاد الزر اعي ـ كلية الزراعة ـ جامعة الزقازيق ـ مصر }
\end{aligned}
$$

\section{Received: 19/04/2018 ; Accepted: 16/05/2018}

الملخص: تعد زريعة الأسماك من أهم مقومات نجاح الاستزر اع السمكي، ومع نجاح تفريخ زريعة أسماك المياه العذبة،

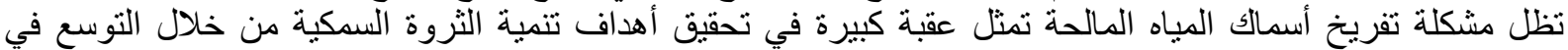

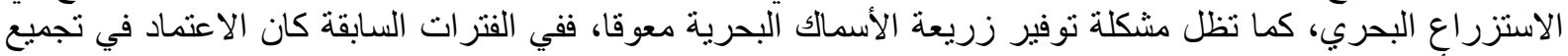

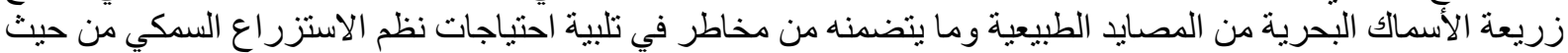

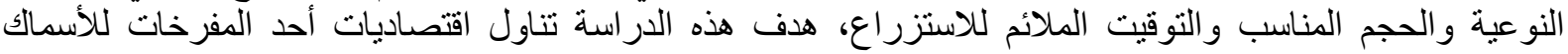

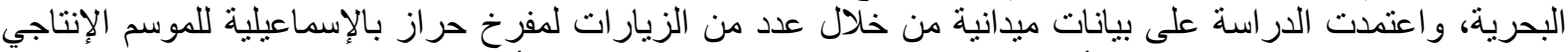

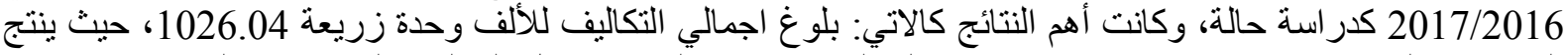

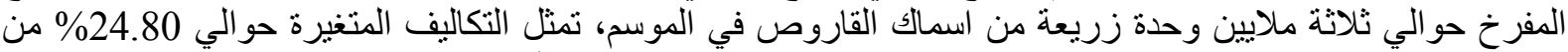

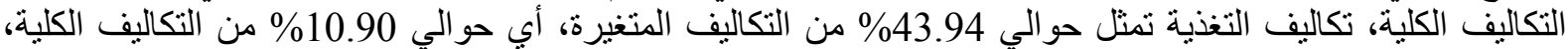
تكاليف الارتيميا تمثل حوالي 9.36\% من التكاليف الكلية، أما التكاليف الثابتة تمثل

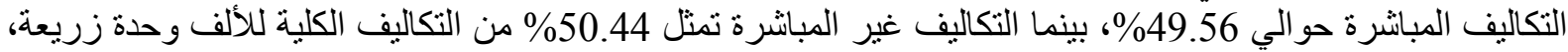

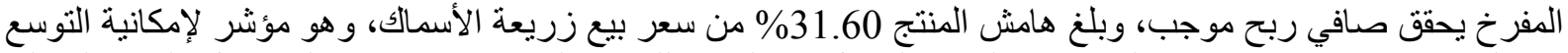

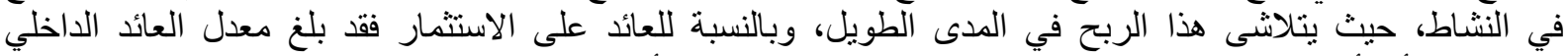

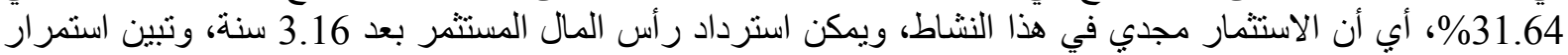

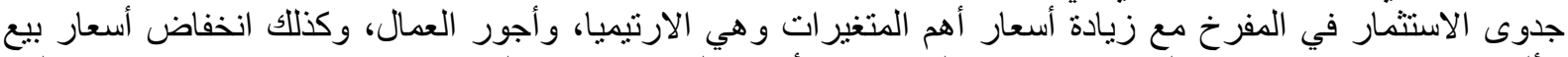

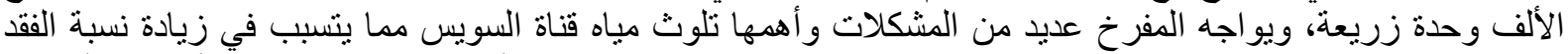

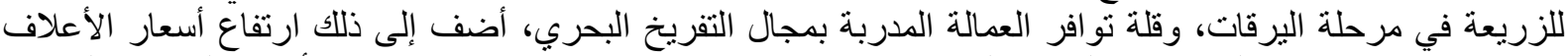

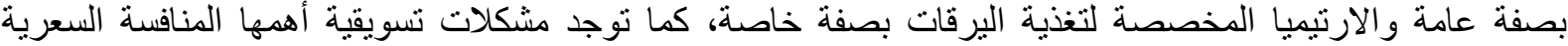
للزريعة التي يتم تجميعها من المصنادر الطبيعية، وأسعار ها من المفرخات الصناعية.

الكلمات الاسترشاديه: اقتصاديات، مفرخ سمكي بحري، حر از ، الإسماعيلية، مصر

واستخدامها في مجالات أخرى، بالإضافة إلى تلوثها

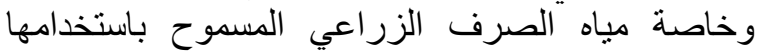

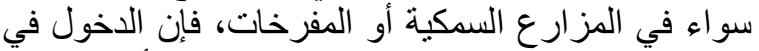

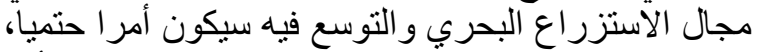

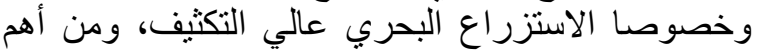

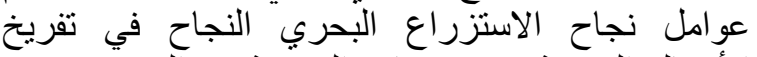

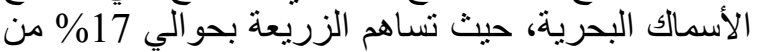

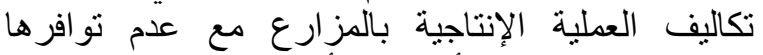

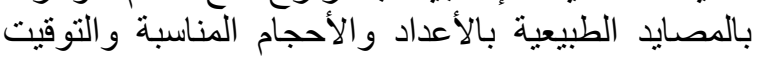

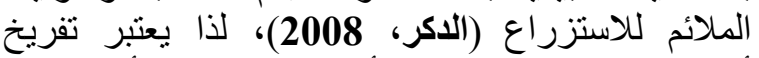

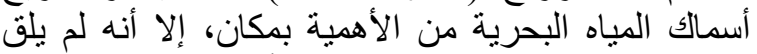

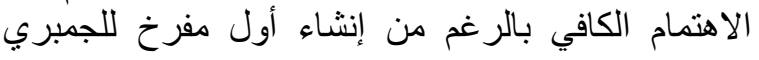

\section{المقدمة والمشكلة البحثية}

يعتبر قطاع الأسماك من القطاعات الهامة لكل من

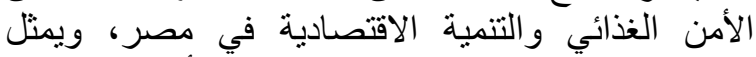

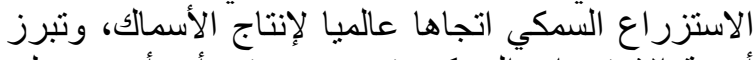

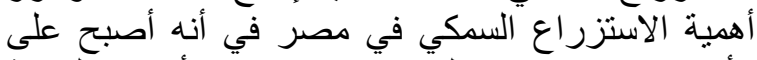

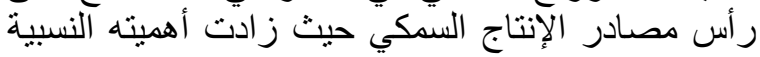

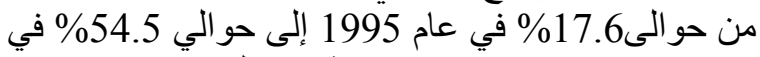

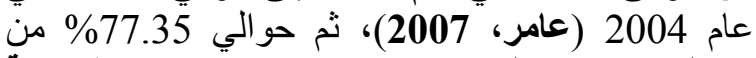
إجمالي الإنتاج السمكي عام 2015 (وزارة الزراعة)

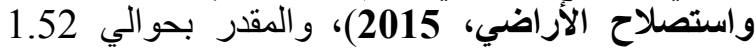
مليون طن. ومع ندرة المياه العذبة المطلوبة للاستزراع

* Corresponding author: Tel. :+201063567069

E-mail address: flyfish_2020@yahoo.com 
بالتوسع في هذا الاتجاه الهام. هــدف البحــــ

يهدف البحث إلى دراسة اقتصاديات المفرخات البحرية بدر اسة حالة لأحد المفرخات المستقرة وذللك من الأنة خلال تحقيق الأهداف الفرعية التالية:

ـ تقدير مؤشرات الكفاءة الاقتصادية للمفرخ البحري.

ـ تقدير مؤشر ات كفاءة الاستثمار للمفرخ البحري. ـ تقدير أثر أهم العوامل الفنية والاقتصادية المؤثرة على فلى كفاءة الاستثمار في المفرخ البحري باستخدام تحليل الحساسية.

ـ اشتقاق المستويات الحرجة (الحدية) لأهم العوامل الفنية

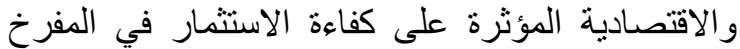
البحري باستخدام أسلوب (Switching value).

\section{مصادر البيانات والطريقة البحثية}

اعتمد البحث بصفة أساسية على بيانات أولية تم تجميعها من مفرخ أهلي وهو مفرخ حراز بالإسماعيلية

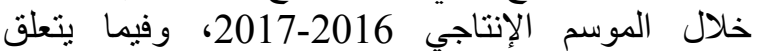
بالطريقة البحثية فقد استند البحث الي اسلوبين تحليليين

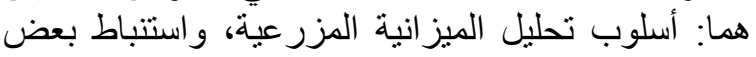

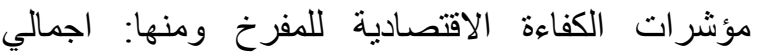

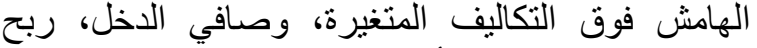
المنتج، هامش المنتج. وأسلوب تحليل جدوى الاستثمار في المشروع وذللك بالاستناد الي أربع معايير وهي البي معدل

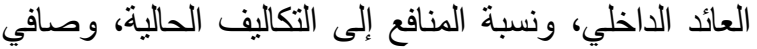

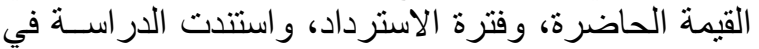

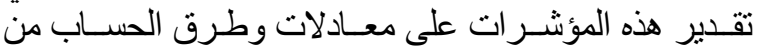

(عامر، Brown, 1979; Ronald, 1981;2016)

\section{النتائج والمناقشتة}

وصف عام للمفرخ

يقع المفرخ بالإسماعيلية طريق البلاح، تم إنشاء

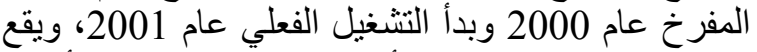

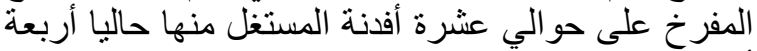

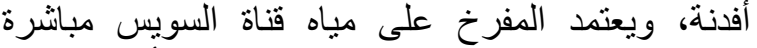

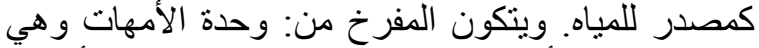

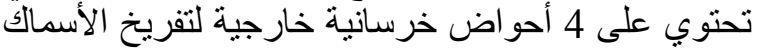

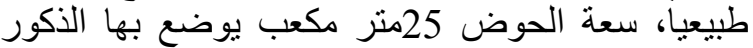

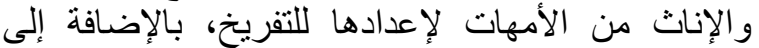

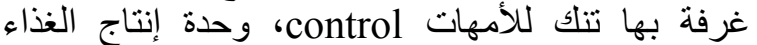

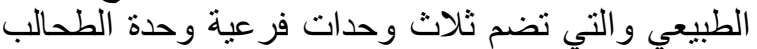
وتتكون من معمل للطحالب تبلغ مساحته 9 منر مربع،
البحري قطاع خاص في عام 1985 بالإسكندرية، ثم نقلت الباع التيري

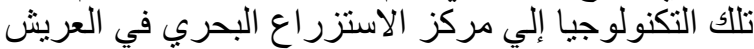

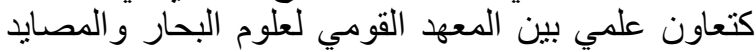

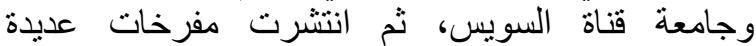
للجمبري البحري في شمال سيناء، و الإسماعيلية، وشرم التئرة

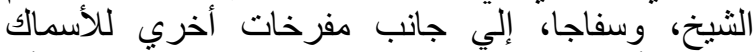

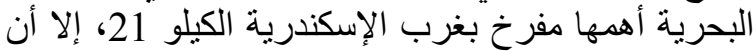

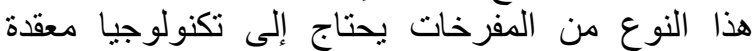

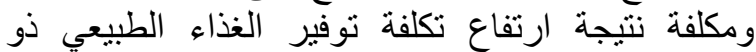

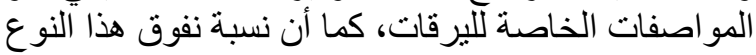

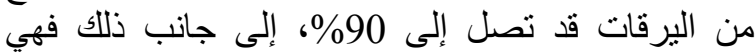

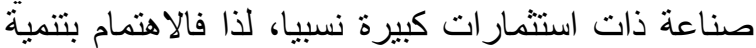

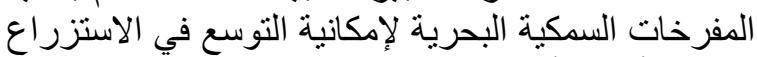

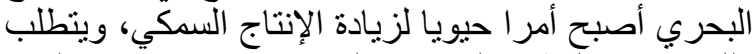

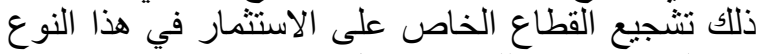

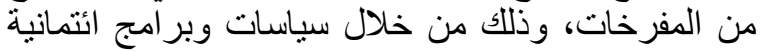

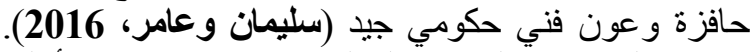
وتساهم الدفرخات البحرية الحالية بنو عيها حكومية وأهية وأهلية

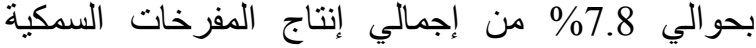

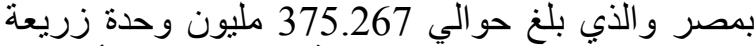
في عام 2015 (وزارة الزراعة واستصلاح الأراضي،

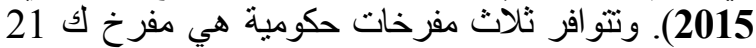

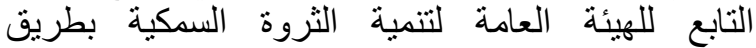

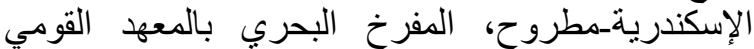

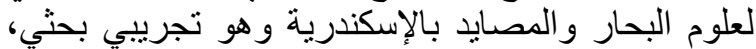

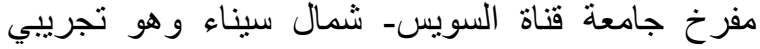

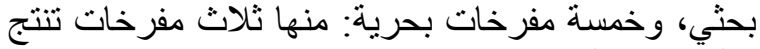

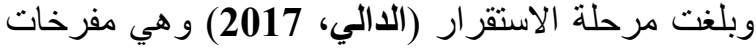

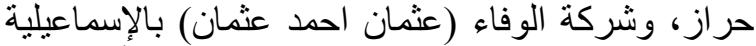

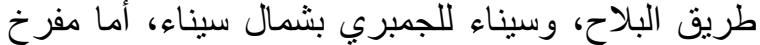

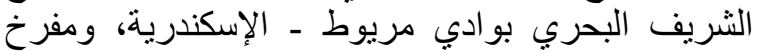

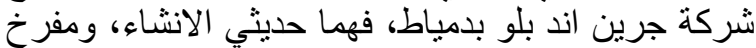
سيفيكو (الشركة الدولية للجمبري و الأسماك) نم إغلاقه منذ النذاءن

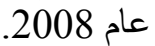

المشكلة البحثية

على الرغم من وجود مصايد بحرية بمصر تبلغ

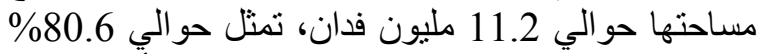

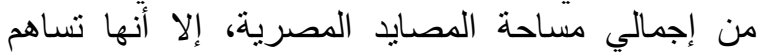
بحوالي 29.9\% من إجمالي انتاج المصايد الطيالي الطبيعية،

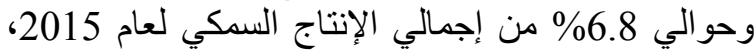

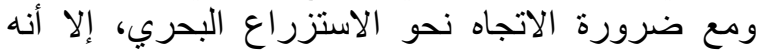

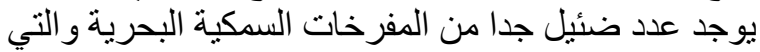

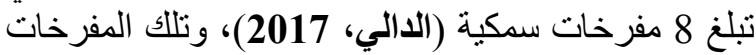

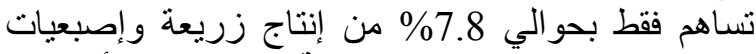

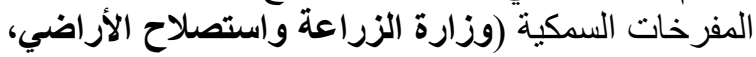

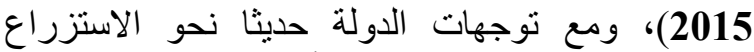

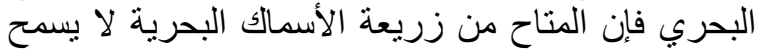


رأس المال وتمثل حو الي 49.66\%، بليها تكاليف الإهلاك

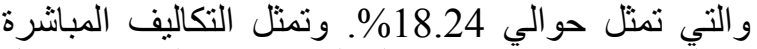
حوالي 56.59\%، بينما التكاليف غير المباشرة تمثل

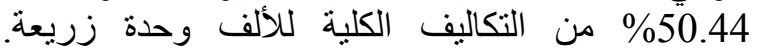
ويوضح جدول 2 إيرادات المفرخ ومؤشرات الات الكفاءة

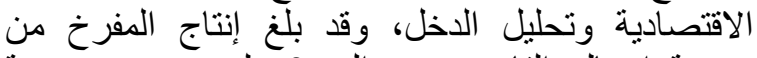

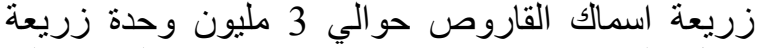
خلال العام الإنتاجي 2016-2017، وقد التراري بلغ إجمالي الإيرادات للمفرخ السمكي حوالي 4.532 مليون جنيها.

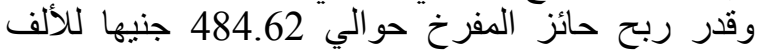
وحدة زريعة. وتثير جميع مؤشرات الكئر الكفاءة الإنتاجية

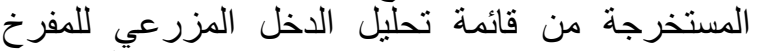

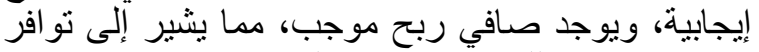

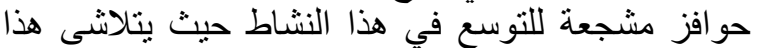
الربح في المدى الطويل. في حين بلغ هامش المفرخ

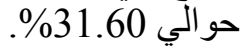

\section{كفاءة الاستثمار للمفرخ}

اعتمدت الدراسة على الفروض التالية لتحليل كفاءة

الاستثمار في المفرخ الأند

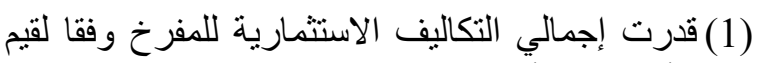
الأصول الر أسمالية، ولقد تضمنت تلإلك التكاليف قيمة الإنة

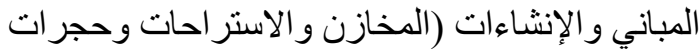

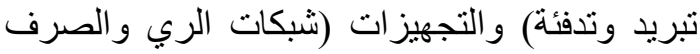

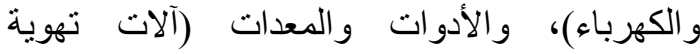

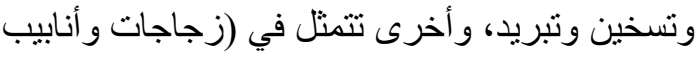
وخر اطيم وسكريات و أدر اج لفرز الأسماك).

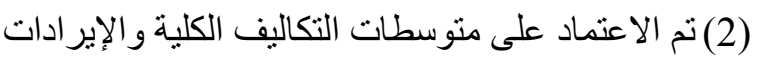

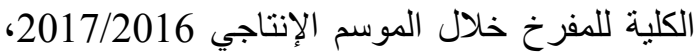

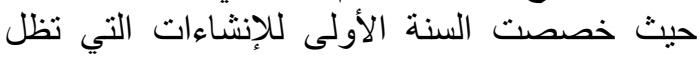

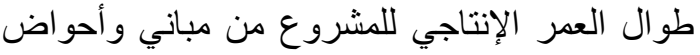

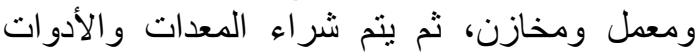

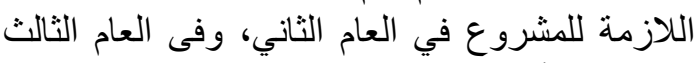
يتم شر اء الأمهات مع بداية عمل المفرخ.

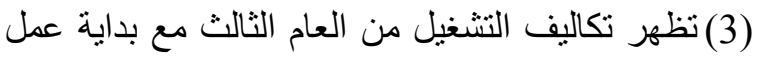
المفرخ، بينما تظهر تكاليف إحلال ثلث القطيع من الفي

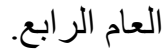

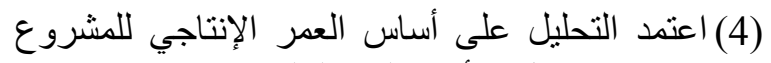
20 سنة تشغيل تبدأ من العام الثالث.

(5) تم استخدام سعر خصم 17.25\% وهو يمثل أفضل فرصة بديلة متاحة لاستثمار رأس المال في المجتمع المعرئ

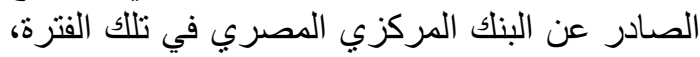

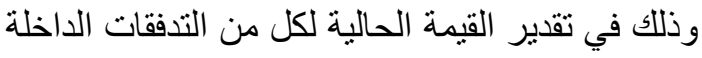
والتدفقات الخارجة طول عمر المشروع.
وفيه يتم استخدام الكيماويات لعمل بيئات مغذية للطحالب إنيات

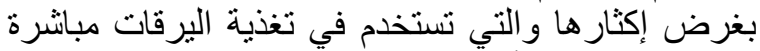

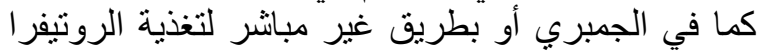

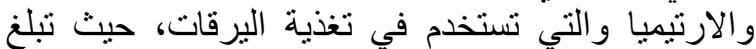

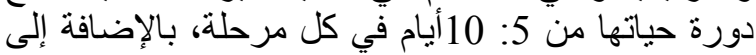

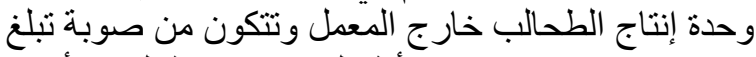

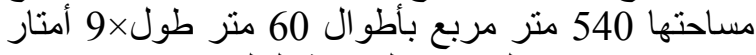

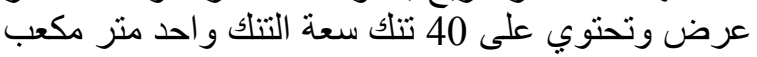

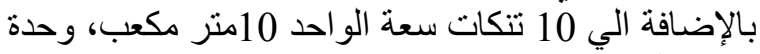

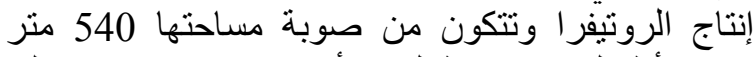

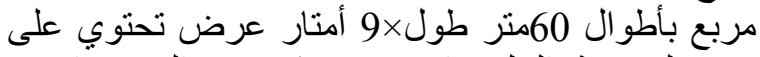

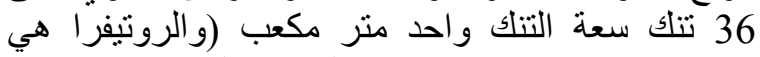

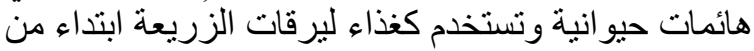

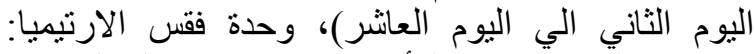

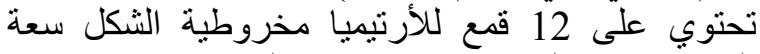

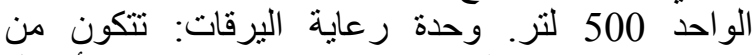

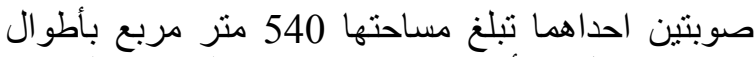

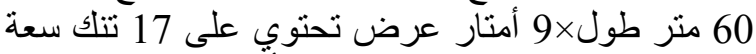

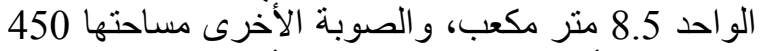

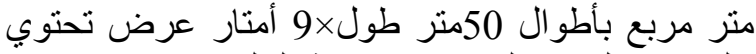

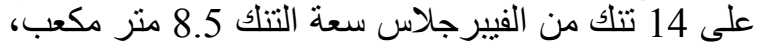
يتم التداخل بين الروتيفرا والارتيميا في التغذية ليرقات التئي

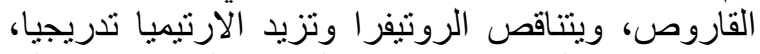

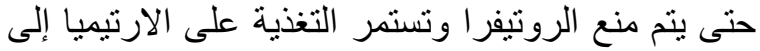

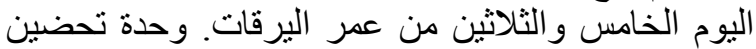
الزريعة وتشمل صوبتين إحداهما تبلغ مساحتهات

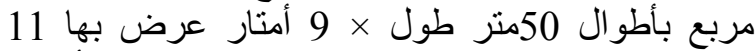

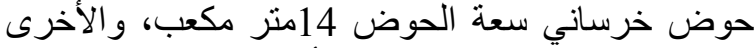

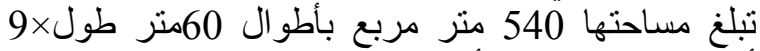

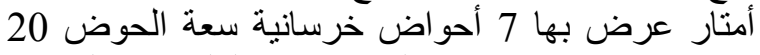

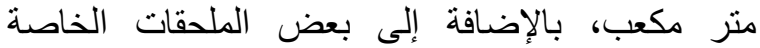
بالمفرخ و التي تتكون من حوض لترفي لترسيب المباه، وحدنين

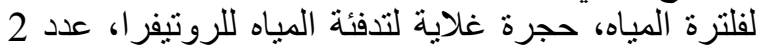

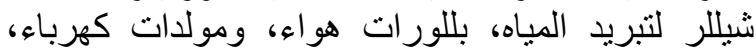
وطلمبات للمباه، مبني إداري، مبلئ هلي سكني للعاملين،

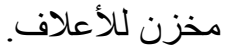

\section{الكفاءة الاقتصادية للمفرخ}

يوضح جدول 1 هيكل التكاليف للمفرخ البحري، ومنه لئه

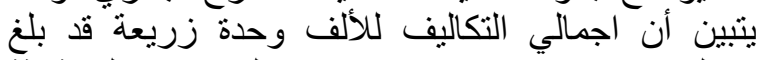

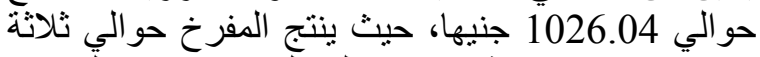

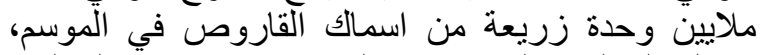

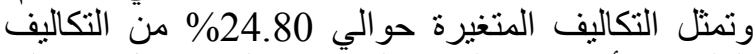

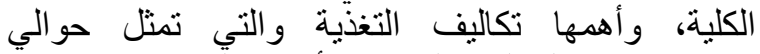

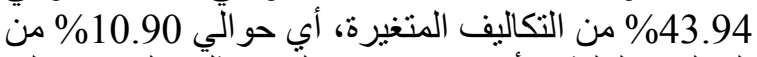
التكاليف الكلية، وأهمها الارتيميا التي تمثل تكلفتها حوالي التي

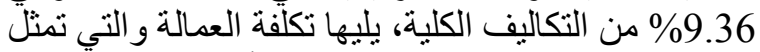
حوالي 7.02\% من \% التكاللف الكلية. أما التكاليف "الثابتة تمثل 75.20\% من التكاليف الكلية، واهمها الفائدة على الئ النية 
جدول 1. هيكل تكاليف تفريخ زريعة الاسماك بالمفرخ السمكي البحري بالإسماعيلية للموسم الإنتاجي 2016-2017 بنود التكاليف

\begin{tabular}{|c|c|c|c|c|}
\hline & & & & 1- تكاليف التغذيفة متغيرة مباشرة \\
\hline 9.36 & 37.73 & 96.00 & 288000.00 & الارتيميا \\
\hline 0.63 & 2.55 & 6.50 & 19500.00 & علف مرحلة اولي \\
\hline 0.58 & 2.36 & 6.00 & 18000.00 & علف مرحلة ثانية \\
\hline 0.32 & 1.30 & 3.30 & 9900.00 & علف مرحلة ثالثة \\
\hline 1.54 & 6.21 & 15.80 & 47400.00 & أعلاف متخصصة \\
\hline 10.90 & 43.94 & 111.80 & 335400.00 & اجمالي تكاليف التغذية \\
\hline 0.78 & 3.14 & 8.00 & 24000.00 & مواد تلدعيم \\
\hline 0.58 & 2.36 & 6.00 & 18000.00 & كيماويات \\
\hline 0.58 & 2.36 & 6.00 & 18000.00 & هرمونات \\
\hline 0.78 & 3.14 & 8.00 & 24000.00 & وقود وزيوت \\
\hline 3.90 & 15.72 & 40.00 & 120000.00 & كهرباء \\
\hline 0.26 & 1.05 & 2.67 & 8000.00 & تكاليف إصلاحات الآلات \\
\hline 7.02 & 28.29 & 72.00 & 216000.00 & 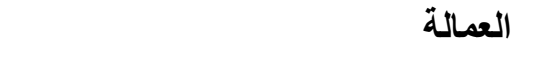 \\
\hline 24.80 & 100.00 & 254.47 & 763400.00 & اجمالي التكاليف المتغيرة المباشرة \\
\hline 0.00 & 0.00 & 0.00 & 0.00 & 2- تكاليف متغيرة غير مباشرة \\
\hline 24.80 & 100.00 & 254.47 & 763400.00 & 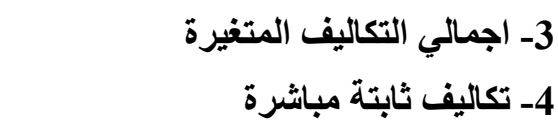 \\
\hline 6.50 & 8.64 & 66.67 & 200000.00 & الصيانة الدورية \\
\hline 0.02 & 0.03 & 0.20 & 600.00 & حق انتفاع \\
\hline 18.24 & 24.26 & 187.17 & 561500.00 & إهلاك الأصول الاستثمارية \\
\hline 24.76 & 32.92 & 254.03 & 762100.00 & 5-تكاليف ثابتة غير مباشرة الثابتة المباشرة \\
\hline 0.78 & 1.04 & 8.00 & 24000.00 & إدارة أصحاب المفرخ \\
\hline 49.66 & 66.04 & 509.54 & 1528630.00 & الفائدة علي راس المال المستثمر \\
\hline 50.44 & 67.08 & 517.54 & 1552630.00 & إجمالي التكاليف الثابتة غير المباشرة \\
\hline 75.20 & 100.00 & 771.58 & 2314730.00 & 6- إجمالي التكاليف الثابتة (4+5) \\
\hline 49.56 & 49.56 & 508.50 & 1525500.00 & 7- إجمالي التكاليف المباشرة (1+4) \\
\hline 50.44 & 50.44 & 517.54 & 1552630.00 & 8- إجمالي التكاليف غير المباشرة (2+5) \\
\hline 100.00 & 100.00 & 1026.04 & 3078130.00 & 9- إجمالي التكاليف الكلية \\
\hline
\end{tabular}


Zagazig J. Agric. Res., Vol. 45 No. (4) 2018

جدول 2. الإير اد ومؤشرات الكفاءة للمفرخ السمكي البحري بالإسماعيلية للموسم الإنتاجي 2016-2017

\begin{tabular}{|c|c|c|c|}
\hline اجمالي القيمة بالجنيه & السعر بالجنيه للوحدة & الكمية & البيان \\
\hline & & & 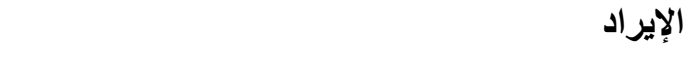 \\
\hline 4500000.00 & 1500 & 3000 & زريعة اسماك القاروص (بالألف وحدة) \\
\hline 16000.00 & 100 & 160 & بيع أمهات مستغني عنها (بالكيلو جرام) \\
\hline 16000.00 & & & صافي التغير في المخزون (قيمة الأمهات) \\
\hline 4532000.00 & & & 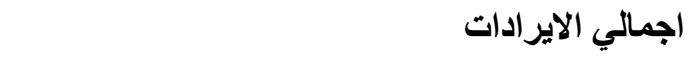 \\
\hline 3768600.00 & & & ي الهامش فوق التكاليف المتغيرة للمفرخ \\
\hline 1256.20 & & & اجمالي الهامش فوق التكاليف المتغيرة للألف وحدة \\
\hline 3006500.00 & & & صافي دخل صاحب المفرخ \\
\hline 1002.17 & & & صافي دخل صاحب المفرخ للألف وحدة \\
\hline 1453870.00 & & & ربح حائز المفرخ \\
\hline 484.62 & & & ربح حائز المفرخ للألف وحدة \\
\hline 31.60 & & & هامش المفرخ (\%) \\
\hline
\end{tabular}

المصدر: جمعت وحسبت من بيانات لمفرخ حراز البحري للموسم الإنتاجي 2016-2017.

تحليل الحساسية للمفرخ البحري يعتبر تحليل الحساسية من أهم المؤشرات التي يمكن التئن

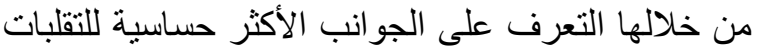

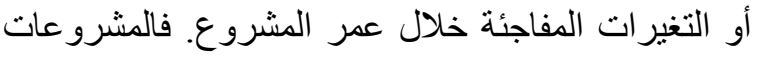
الزراعية بصفة عامة والاستزر اعلئ السمكي بصفة النفة خاصنة

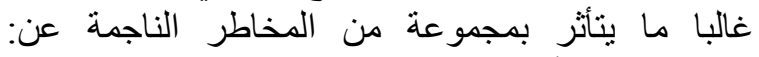

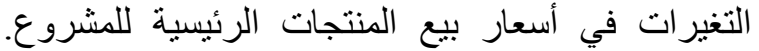

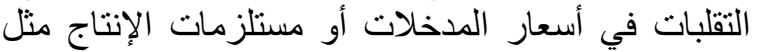

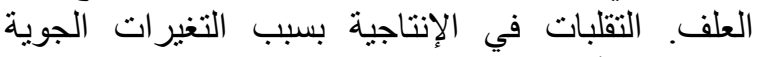

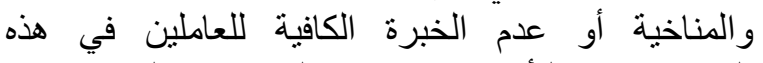

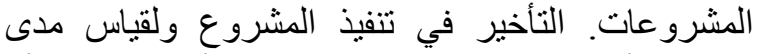

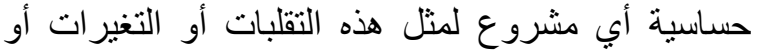

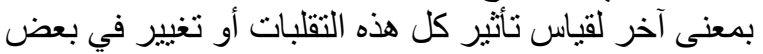

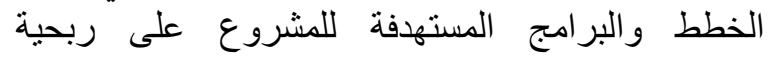
المشروع، فقد تم إجراء تحليل الحساسية في الحالات الحتئ

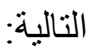

1- أثر زيادة سعر الارتيميا بمقدار 10\% على جدوى الاستثمار للمفرخ البحري لأنها تمثل حوالي

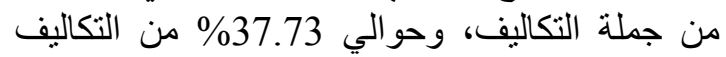
المتغيرة، وتبين من جدول 6 أن زيادة سعر الارتيميا
(6) تم حساب قيمة الخردة للأصول الرأسمالية للمفرخ

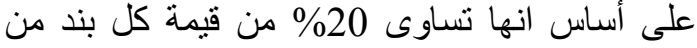
بنود التكاليف الاستثمارية ما عدا أمهات الأسماك، فقد فئ

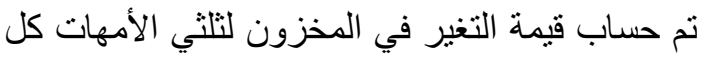

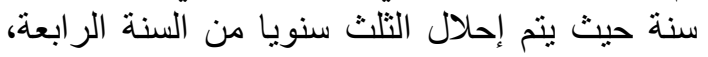
كما هو موضح بجدول 3.

(7) تم تصميم جدول التدفقات النقدية الداخلة والخارجة و الصافية طوال عمر المشروع الإنتاجي، تضدنت التدفقات الخارجة كل مصروفات المنات المشروع (التكاليف

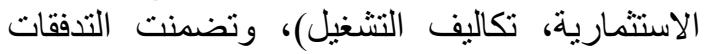
الداخلة كل عو ائد المشروع (عائد بيع زريعة أسماك ولئك القاروص و اسماك الأمهات الكبيرة التي ينت التم احلالها

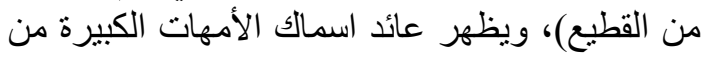
العام الر ابع مع بداية إحلال القطيع.

تثير بيانات جدول 5 لمؤشر ات فياس كفاءة الاستثمار الي جدوى الاستثمار في هذا النشاط، و إمكانية استرداد رأس المال المستثر في المشروع في أقل من من ثلاث

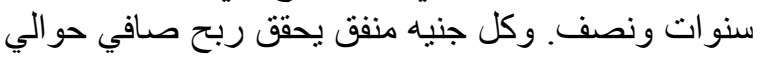
57 قرشا، كما أن عائد الاستثمار أعلي من أعلى سلي سعر

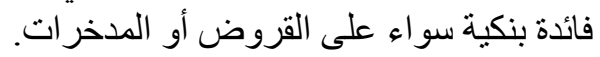


جدول 3. هيكل التكاليف الاسنثمارية للمفرخ

\begin{tabular}{|c|c|c|c|c|}
\hline الخردة & الإنتاجي & الثراء & الثراء & الأصل \\
\hline & & & & المباني لأقَسام الأمهات و الزريعة والتحضين ومعمل الطحالب \\
\hline 0 & 20 & 4000000 & 1 & 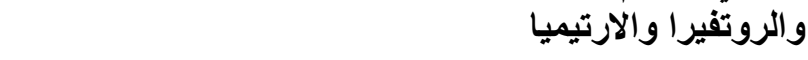 \\
\hline 0 & 20 & 150000 & 1 & تجهيز الموقع \\
\hline 0 & 20 & 400000 & 1 & 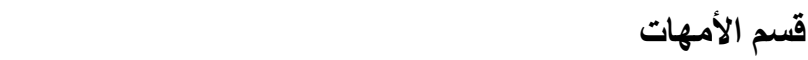 \\
\hline 0 & 20 & 120000 & 1 & قسم التقريخ \\
\hline 0 & 20 & 341000 & 1 & قسم رعاية اليرقات \\
\hline 0 & 20 & 144000 & 1 & 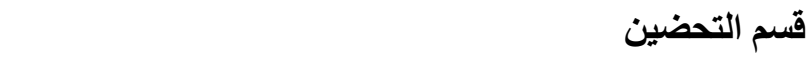 \\
\hline 0 & 20 & 40000 & 1 & 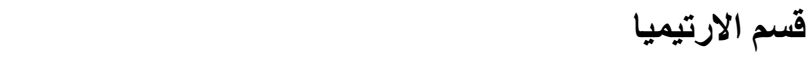 \\
\hline 0 & 20 & 120000 & 1 & قسم الطحالب (المعمل) \\
\hline 0 & 20 & 312000 & 1 & الطحالب الخارجية \\
\hline 0 & 20 & 160000 & 1 & قسم الروتفيرا \\
\hline 72000 & 5 & 360000 & 2 & مولد كهربائي \\
\hline 48000 & 5 & 240000 & 2 & طلمبات \\
\hline 32000 & 5 & 160000 & 2 & بلورات \\
\hline 80000 & 5 & 400000 & 2 & وحدات فلاتر و UV \\
\hline 0 & 20 & 700000 & 1 & شبكة ري وصرف \\
\hline 0 & 20 & 175000 & 1 & شبكة تهوية \\
\hline 0 & 20 & 180000 & 1 & شبكات كهرباء \\
\hline 0 & 20 & 180000 & 1 & مخازن وحجرات تبريد وتدفئة \\
\hline 0 & 20 & 400000 & 1 & مباني إدارية وسكنية \\
\hline \multirow[t]{2}{*}{6000} & 5 & 30000 & 2 & أخرى \\
\hline & & 8612000 & & اجمالي التكاليف الاستثمارية \\
\hline
\end{tabular}


جلول 4. قائمة التدفقات النقدية لمفرخ حراز البحري خلال عمر المشروع

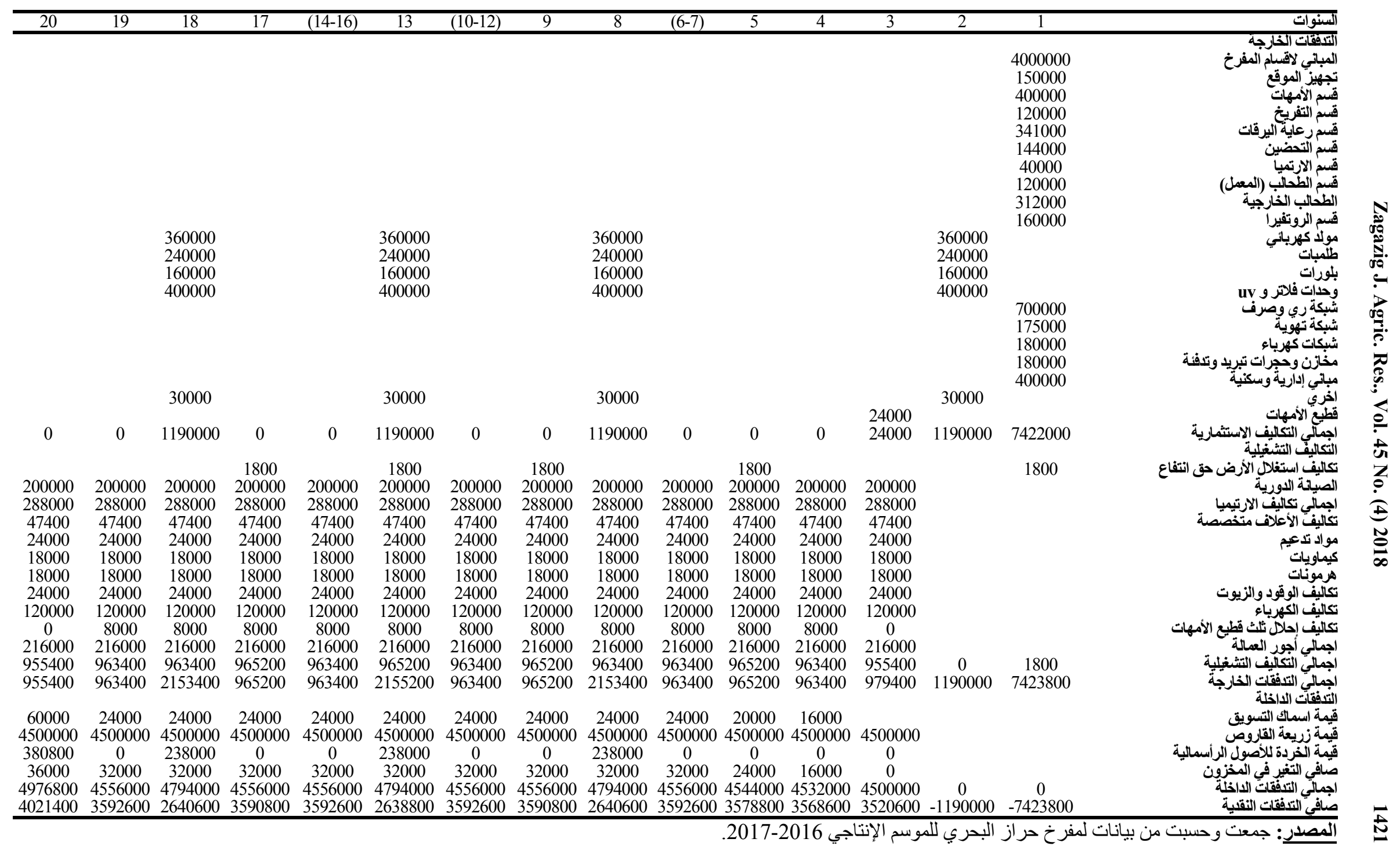


جدول 5. مؤشرات كفاءة الاستثمار للمفرخ للعام الإتتاجي 2016-2017

\begin{tabular}{|c|c|c|}
\hline المفرخ البحري & الوحدة & المؤشـــــــر \\
\hline 31.64 & $(\%)$ & معدل العائد الداخلي \\
\hline 1.57 & جنيها & نسبة المنافع الى التكاليف \\
\hline 6.811 .369 & جنيها & صافي القيمة الحاضرة \\
\hline 3.16 & سنة & فترة الاسترداد \\
\hline
\end{tabular}

المصدر: حسبت من قائمة التدفقات السنوية.

أدت إلى انخفاض معدل العائد الداخلي من 31.64\% \%

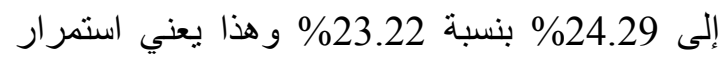

جدوى الاستثمار للمشروع.

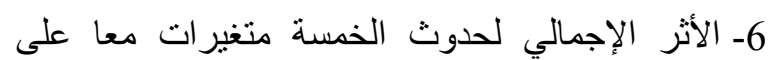

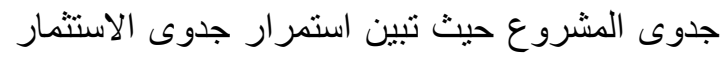

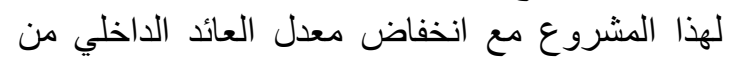
31.64\% إلى 18.64\% بنسبة \% \% \% \%

اشتقاق المستويات الحدية (الحرجة) من المتغيرات الهامة المؤثرة على كفاعة المفرخات السمكية المية المن المغية

من بيانات جدول 6 أمكن تحديد المستويات الحرجة

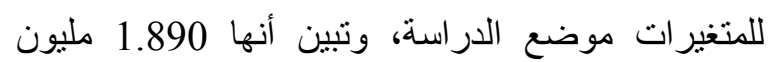
وحدة، و945 جنيها سعر البيع للألف وحدة زبنة زريعة،

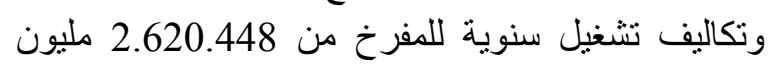

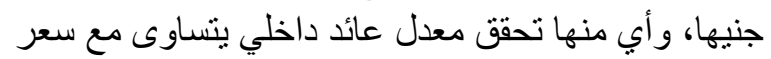

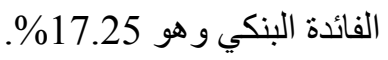

\section{المشكلات التي تواجه المفرخ}

يواجه المفرخ عديد من المشكلات منها الإنتاجية

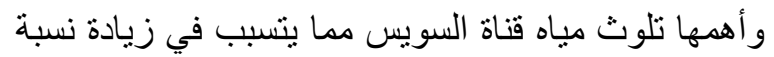

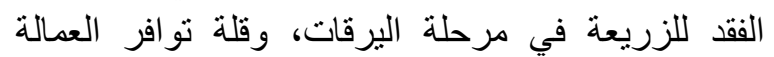

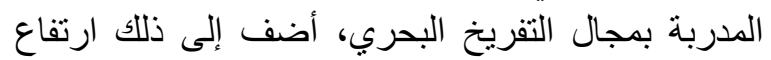

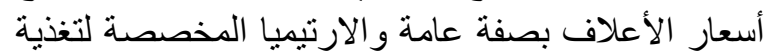

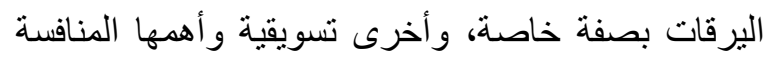

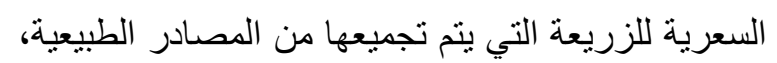
و أسعار ها من المفرخات الصناعية لتصنية.
من 1600 جنية للطن إلى 1760 جنية للطن أدى إلى الى

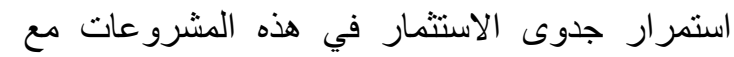
انخفاض معدل العائد الداخلي من هن هـ

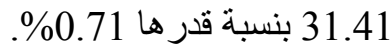

2- أثر زيادة أجور العمالة الموسمية المستأجرة بمقدار

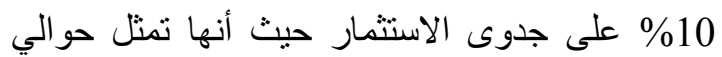

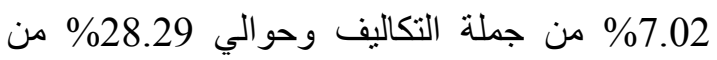

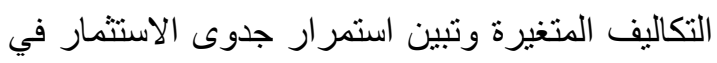
هذه المشروعات مع انخفاض معدل العائد الداخلي من الفئ

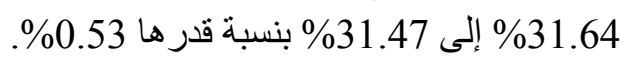

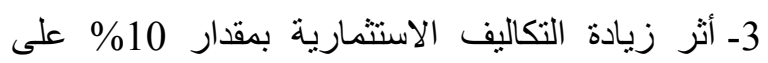

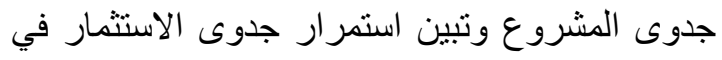

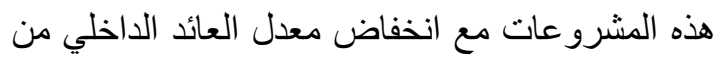

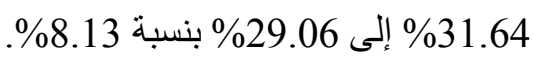

4- أثر انخفاض أسعار بيع زريعة الأسماك بنسبة 10\%

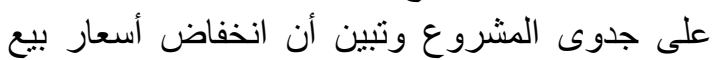

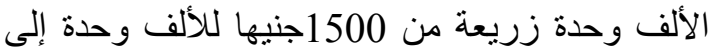

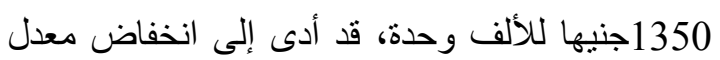

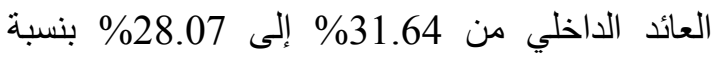

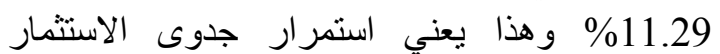
ل كشروع. 5- أثر انخفاض الإنتاج من زريعة الأسماك بنسبة 20\%

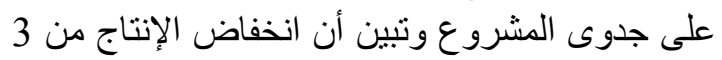

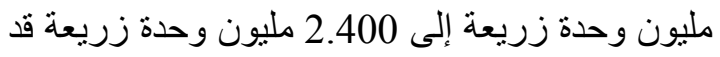


Zagazig J. Agric. Res., Vol. 45 No. (4) 2018

1423

جدول 6. أثر التغيرات غير المرغوبة للمشروع على مؤشرات كفاءة الاستثمار للمفرخ للعام الإنتاجي 2016-2017

\begin{tabular}{|c|c|c|c|c|c|}
\hline نسبة التغير (\%) & 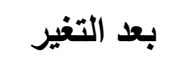 & الوضع الحالي & 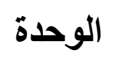 & المؤشر & 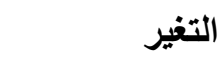 \\
\hline $0.71-$ & 31.41 & 31.64 & $(\%)$ & معدل العائد الاخاخلي & \\
\hline $0.98-$ & 1.55 & 1.57 & جنيها & نسبة المنافع الحالية إلى التكاليف الحالية & زيادة سعر \\
\hline $1.71-$ & $6,694,944$ & 6.811 .369 & جنيها & صافي القيمة الحاضرة & الارتيميا 10\% \\
\hline 0.71 & 3.18 & 3.16 & 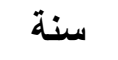 & فترة الاسترداد & \\
\hline $0.53-$ & 31.47 & 31.64 & $(\%)$ & معدل العائد الاخاخلي & \\
\hline $0.74-$ & 1.56 & 1.57 & جنيها & نسبة المنافع الحالية إلى التكاليف الحالية & زيادة أجور \\
\hline $1.28-$ & $6,724,050$ & 6.811 .369 & جنيها & صافي القيمة الحاضرة & العمالة 10\% \\
\hline 0.53 & 3.18 & 3.16 & 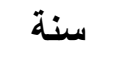 & فترة الاسترداد & \\
\hline $8.13-$ & 29.06 & 31.64 & $(\%)$ & معدل العائد الداخلي & \\
\hline $6.27-$ & 1.47 & 1.57 & جنيها & نسبة المنافع الحالية إلى التكاليف الحالية & زيادة التكاليف \\
\hline $11.42-$ & $6,033,683$ & 6.811 .369 & جنيها & صافي القيمة الحاضرة & $\% 10$ \\
\hline 8.85 & 3.44 & 3.16 & 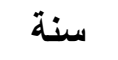 & فترة الاسترداد & \\
\hline 11.29- & 28.07 & 31.64 & $(\%)$ & معدل العائد الداخلي & \\
\hline $9.84-$ & 1.42 & 1.57 & جنيها & نسبة المنافع الحالية إلى التكاليف الحالية & انخفاض أسعار \\
\hline $26.71-$ & $4,992,220$ & 6.811 .369 & جنيها & صافي القيمة الحاضرة & الأسمالك 10\% \\
\hline 12.72 & 3.56 & 3.16 & 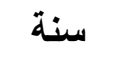 & فترة الاسترداد & \\
\hline $23.22-$ & 24.29 & 31.64 & $(\%)$ & معدل العائد الداخلي & \\
\hline $19.67-$ & 1.26 & 1.57 & جنيها & نسبة المنافع الحالية إلى التكاليف الحالية & ا انخفاض الإنتاج \\
\hline $53.42-$ & $3,173,072$ & 6.811 .369 & جنيها & صافي القيمة الحاضرة & الأسمالك 20\% \\
\hline 30.25 & 4.12 & 3.16 & 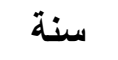 & فترة الاسترداد & \\
\hline 41.09- & 18.64 & 31.64 & $(\%)$ & معدل العائد الداخلي & \\
\hline $33.16-$ & 1.05 & 1.57 & جنيها & نسبة المنافع الحالية إلى التكاليف الحالية & 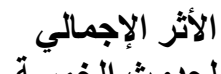 \\
\hline 89.19- & 736,321 & 6.811 .369 & جنيها & صافي القيمة الحاضرة & 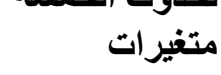 \\
\hline 69.76 & 5.37 & 3.16 & 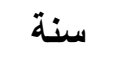 & فترة الاسترداد & \\
\hline
\end{tabular}




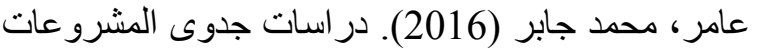
الزراعية، الطبعة الأولي، المهنس للطباعة، الزقازيق،

وزارة الزراعة واستصلاح الأراضي (2015). الهيئة

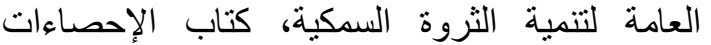

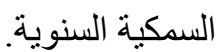

Brown, M.L. (1979). Farm Budget from Income Analysis To Agricultural Project Analysis. $1^{\text {ST }}$ Ed., Johns Hopkins Univ. Press, Baltimore and London.

Ronald, D.K. (1981). Farm Management Planning, Control and Implementation. Int. Student Ed. McGraw-Hill International Book Company, Auckland London, Inc., Tokyo Japan.

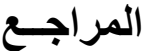

الدالي، نسمة منصور أحمد علي (2017). التقييم

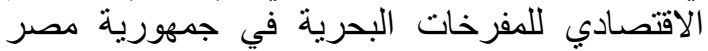

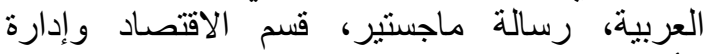
الأعمال الزراعية، كلية الزراعة، جامعة الإسكندرية.

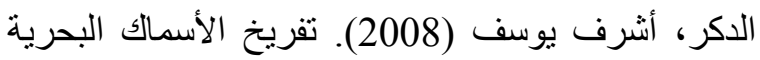

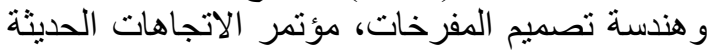

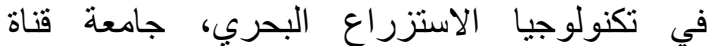
السويس، 27-2 إلى 1-3، العريش.

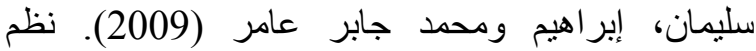
الاستزر اع السكيـ الإدارة و الاقتصاديات، دار الفكر

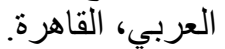
عامر، محمد جابر (2007). الإنتاج السمكي في مصر، الزئ كتاب المؤتمر الخامس عشر للاقتصاديين الزراعيين،

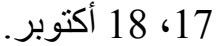




\title{
AN ECONOMIC STUDY OF MARINE FISH HATCHERIES IN EGYPT: A CASE STUDY ON HERAZ HATCHERY IN ISMAILIA
}

\author{
Rasha A.A. Naiel ${ }^{1}$, M.G.M. Amer², A.A. Ibrahim ${ }^{2}$ and M.M.Soliman ${ }^{1}$ \\ 1. Inst. Agric. Econ. Res., Agric. Res. Cent., Egypt \\ 2. Dept. Agric. Econ., Fac. Agric., Zagazig Univ., Egypt
}

\begin{abstract}
Fish fry is one of the most important factors for the success of fish aquaculture, and with the success of the hatching of freshwater fish. The problem of hatching of saltwater fish remains a major obstacle to the achievement of fisheries development objectives through the expansion of marine aquaculture. The problem of providing marine fish fry remains impeded. In previous periods, reliance was placed on the collection of marine fish fry from capture fisheries and the risks involved in meeting the needs of the fish farming systems in terms of quality, size and appropriate timing for aquaculture. The objective of this study is to address the economics of a hatchery for marine fish in order to expand these hatcheries to meet the future needs of this promising fish culture. The study was based on field data through a number of visits to Heraz hatchery in Ismailia Governorate for the production season 2016/2017 as a case study. The main results are as follows: The total cost is estimated at 1026.04 LE per thousand of fry unit. The hatchery produces 3 millions of fry unit in season from European seabass fish. The variable costs is estimated at $24.80 \%$ of the total costs. The feed costs is estimated at $43.94 \%$ of the variable costs and estimated at $10.90 \%$ of the total costs. Artemia costs is estimated at $9.36 \%$ of the total costs. The fixed costs is estimated at $75.20 \%$ of the total costs. The direct and indirect costs is estimated at $49.56 \%$ and $50.44 \%$ of the total costs of the thousand of fry unit. The hatchery achieves positive net profit, and the producer margin reached $31.60 \%$ of the selling price of thousand units, which is an indicator of the possibility of expansion of activity, where this profit will disappeared in the long term. In terms of return on investment, the internal rate of return was $31.64 \%$, meaning that the investment is viable in this activity. The payback period can be recovered after 3.16 years. The continued feasibility of investment in the hatchery with the increase in the prices of the most important variables are Artemia, and wages of workers, as well as lower prices for the sale of one thousand units of fry. The hatchery faces many problems, the most important of which is the pollution of the Suez Canal water, which leads to an increase in the loss of fry in the larvae stage. Moreover, there is no available trained labor in the field of marine hatching. In addition to the increase in feed prices in general and in particular larvae for feeding larvae. There are also marketing problems, the most important of which is the price competition for fry collected from natural sources and prices of industrial hatcheries.
\end{abstract}

Key words: Economics, marine fish hatchery, heraz, Ismailia, Egypt.

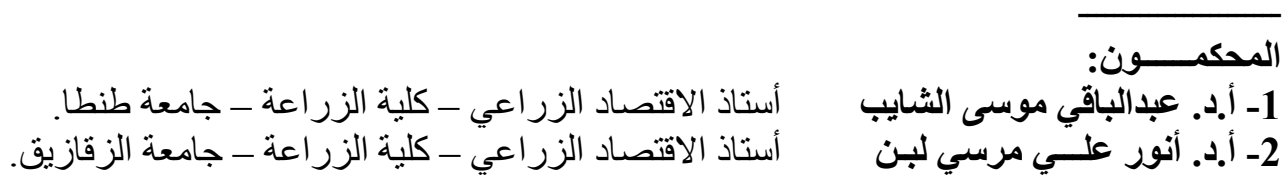

